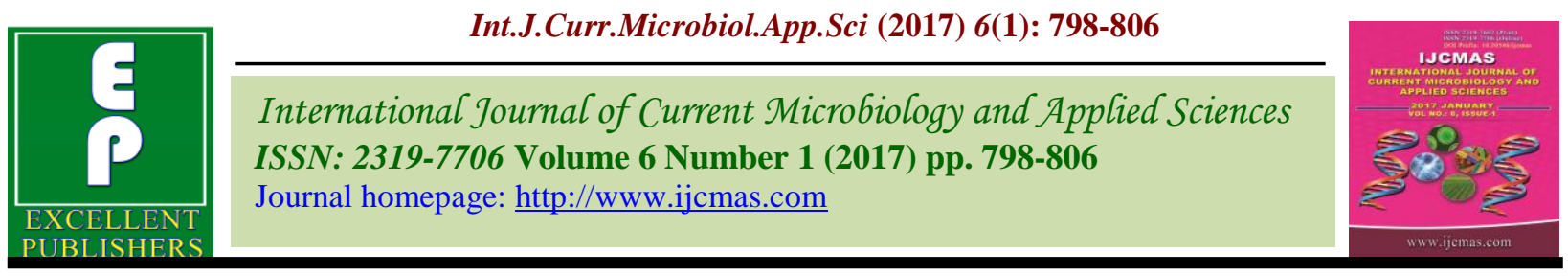

Original Research Article

http://dx.doi.org/10.20546/ijcmas.2017.601.094

\title{
Isolation and Characterization of Native Isolates of Bacillus thuringiensis from Vidarbha Region
}

\section{Dipika Ashokrao Padole ${ }^{1^{*}}$, Mangesh Pradip Moharil ${ }^{1}$, Krishnananda Pralhad Ingle ${ }^{1}$ and Shyamkant Munje ${ }^{2}$}

\author{
${ }^{1}$ Biotechnology Centre, Dr. Panjabrao Deshmukh Krishi Vidyapeeth, Akola, \\ Maharashtra, India \\ ${ }^{2}$ Regional Research Centre for Soybean, Amaravati, Dr. Panjabrao Deshmukh Krishi \\ Vidyapeeth, Maharashtra, India
}

*Corresponding author

\section{A B S T R A C T}

Keywords

Bacillus thuringiensis, IMViC, Phase contrast microscopy, Btk HD-1 - Bacillus thuringiensis var. kurstaki HD-1, Malachite green

Article Info

Accepted:

29 December 2016

Available Online:

10 January 2017
The present investigation was undertaken for the isolation and characterization of B. thuringiensis strains from different habitats based on the morphology, staining properties and biochemical characters. Fifty four samples including soil samples (30), phylloplane (21), insect cadaver (2) and stored grain dusts (1) were collected from cultivated and barren lands not having Bt history. Total $154 \mathrm{Bt}$ isolates obtained from different sources comprising 89, 59 and 6 from soil, phylloplane and insect cadaver respectively, whereas, none of the single isolate was obtained from seed dust. The local $B t$ isolates showed positive morphological characters as that of standard strain Bacillus thuringiensis kurstaki HD -1 (Btk HD-1). Phase contrast microscopic evaluation showed that, more than $75 \%$ of the total 154 isolates lead significant resemblance with standard strain. Further characterization of the probable native $B t$ isolates was carried out biochemically with different tests such as IMViC tests and for the presence of enzymes like catalase, urease, and nitrate reductase utilization which showed that $70-80 \%$ of the total probable $B t$ isolates has positive characters as standard strain. $B t$ isolation index suggested that soil had highest background $B t$ population followed by insect cadaver and leaves.

\section{Introduction}

The indiscriminate use of chemical insecticides leads considerable damage to environment and imbalance the ecology. The continuous and overuse of chemical insecticides imparts resistance in vectors of human diseases along with agricultural pests (Georghiou, 1990).
Biocontrol is the better option than chemical pesticides in order to control insect pests in insect management programme (Aramideh $e t$ al., 2010). Bacillus thuringiensis is gram positive, aerobic, endospore forming and ubiquitous in nature with genome size 2.4 to 5.7 Mbp, distinguished from other bacilli by 
its production of parasporal crystal protein (delta-endotoxin) (Yamamoto and Powell,1993). Under alkaline condition this crystal protein gets solubalized in insect midgut and then activated by intrinsic proteases into an active toxin which selectively binds to specific receptor in the cell membrane, leading to pore formation and ultimately insect death (Soberón et al., 2000; Eswarapriya et al., 2010).

Bacillus thuringiensis has been successfully used as bio-insecticide over the last 60 years, or in transgenic development since $19^{\text {th }}$ century because of its high specificity, safety and effectiveness against wide spectrum of human disease vectors and agricultural pests (Schnepf et al., 1998; Nester et al., 2002). The growing public concern, environmental regulations and buildup of resistant biotypes to synthetics insecticides have leads increased interest in alternative environment-friendly insect management and control strategies. Thus B. thuringiensis could offer an alternative to chemical insecticides. Keeping this view under consideration, the present study deals with the isolation and characterization of $B t$ isolates from its native ecological nitche from vidarbha region of Maharashtra for the identification of candidate gene which could be incorporated into pest control program.

\section{Materials and Methods}

\section{Site of Sample Collection/Sample collection}

Collection of soil, Leaf, seed dust and insect cadaver samples was done according to the method given by Asokan and Puttaswamy, (2007) with necessary modifications from the locations with as diverse plantation as possible either from cultivated or from non cultivated fields without having Bt history, neither sown nor spread.
Soil- One gram of soil samples were obtained by scrapping off surface material up to $2-5 \mathrm{~cm}$ below the surface with sterile spatula from 11 locations ranging from agronomical, horticultural, medicinal plantation to barren land. These samples were stored in sterile polythene bags until further use.

Leaves- Three leaves each from above mentioned plantation were collected and processed immediately to isolate Bacillus thuringiensis.

Insect Cadaver- Two dead and diseased lepidopteron larvae obtained from Gram and Cotton plantation were collected in sterile vials and stored at $4{ }^{0} \mathrm{C}$ until further use.

Seed Dust- One gram of grain dust from Sorghum was collected from Central Research Station Godown, Dr. PDKV, Akola and stored in sterile polythene bags until further use.

\section{Isolation of $B t$ from the samples}

Isolation of Bacillus thuringiensis from soil, leaf, insect cadaver and seed dust was done according to the method given by Asokan and Puttaswamy (2007) where one gram of soil was subjected to acetate selection. Acetate leads inhibition of $B t$ spores germination and non spore forming bacteria were eliminated by heat treatment. After acetate selection, 100 to 300 spore forming colonies were recovered per plate (Martin and Travers, 1989).

\section{Characterization of native isolates of $B t$ isolates}

Morphological characterization- All the colonies grown on the LB agar showing similarities with that of standard strain $B t \mathrm{k}$ HD-1 was grown on LB agar for $24 \mathrm{hrs}$ at $30^{\circ} \mathrm{C}$ and observed for colony morphology under stereoscopic zoom microscope. 
Colonies were observed for colony colour, colony shape, colony margin, colony elevation and colony surface.

Characterization based on staining- Twenty four hrs old bacterial cultures were used to observe the morphology of vegetative cells and differential characterization of probable $B t$ isolates by using gram staining, spore staining with malachite green and amido black (Allwin et al., 2007), crystal staining with CBB-G 250 (Kati et al., 2007) and sporulated cultures were used for phase contrast microscopy and observed under compound microscope using $\mathrm{Ph} 2$ objective lens for the presence or absence of crystals.

\section{Biochemical Characterization}

Characterization of probable $\mathrm{Bt}$ isolates based on staining were further confirmed by the biochemical characterization which comprises of IMViC test (indole production, methyl red, Voges-Proskauer, citrate utilization test). Enzymes like catalase, urease, and nitrate reductase were studied (Sarita Agrahari et al. 2008).

\section{Phase contrast microscopy}

$B t$ colonies were suspended in sterile distilled water as a wet mount and examined with phase contrast microscope (100X) for the presence of parasporal crystals bodies. Colonies were then classified into different groups based on their crystal shape (Lopez Pazos et al., 2007). Potential Bt candidates were sub-cultured and purified on nutrient agar plates and then stored as stock culture in sterile liquid Nutrient Broth containing 50\% glycerol at $-20^{\circ} \mathrm{C}$ (Hernandez et al., 2005).

\section{Results and Discussion}

Due to ubiquitous nature of $B t$, samples were collected from different sources such as soil, seed dust, phylloplane and insect cadaver from university fields, Dr. PDKV, Akola in order to isolate it from native ecological niche. Thirty, twenty one, two and one samples were collected from soil, phylloplane, insect cadaver and seed dust, respectively. With respect to the number of probable $B t$ isolate obtained from different sources, a highest number of eighty nine isolates were obtained from soil, fifty nine from phylloplane, six from insect cadaver and not a single isolate was obtained from seed dust sample. The number of probable $B t$ isolates showed that $B t$ was positively ubiquitous in native ecology of university fields. Table 1 gives an idea about number of isolates obtained from the samples taken from different sources, whereas, figure 1 depicts isolated colonies of native $B t$ isolates.

In India, similar results were obtained by Asokan and Puttaswamy (2007) and Sarita Agrahari et al., (2008) when isolated $B t$ from soil, leaf, seed dust and insect cadaver respectively.

All the isolates obtained from different sources were morphologically characterized by studying the different characters such as colony colour, colony elevation, colony margin, colony shape and colony surface. Bacillus thuringiensis var. kurstaki HD-1 was used as a positive standard having morphological characters as flat, circular, creamy white colour colony with irregular margin and wavy surface (figure 2). Based on these characters, seventy seven out of eighty nine, thirty six out of fifty nine, and all isolates out of six from soil, leaf, insect cadaver respectively were classified as probable $B t$ isolates. Table 2 describes the morphological characterization of the representative $B t$ isolates and figure 2 represents $B t$ isolates obtained on the basis of morphological characterization. 
Kaur et al., (2006), Kati et al., (2007) and Sarita Agrahari et al., (2008) done similar work to characterize $B t$ isolates on morphological basis.

The biochemical characterization of representative $B t$ isolates was carried out for catalase, urease, indole, nitrate reductase and citrate test. Standard Btk HD-1 showed positive reaction for catalase, urease, nitrate reduction and MRVP test while negative for indole and citrate utilization test. It is depicted in table 3 and figure 3.

Table.1 Isolation of $B$. thuringiensis from different habitats

\begin{tabular}{|l|l|l|l|c|c|c|}
\hline SN & \multicolumn{1}{|c|}{ Particulars } & Soil & Leaf & $\begin{array}{c}\text { Insect } \\
\text { Cadaver }\end{array}$ & Seed Dust & Total \\
\hline 1 & Samples Collected & 30 & 21 & 2 & 1 & 54 \\
\hline 2 & No. of probable $B t$ isolates & 89 & 59 & 6 & 0 & 154 \\
\hline
\end{tabular}

Table.2 Morphological characterization of representative isolates of B. thuringiensis

\begin{tabular}{|c|c|c|c|c|c|c|}
\hline \multirow[b]{2}{*}{ Sr No. } & \multirow[b]{2}{*}{ Isolate No. } & \multicolumn{5}{|c|}{ Morphological Characteristics } \\
\hline & & $\begin{array}{l}\text { Colony } \\
\text { Colour }\end{array}$ & $\begin{array}{l}\text { Colony } \\
\text { Shape }\end{array}$ & $\begin{array}{l}\text { Colony } \\
\text { Margin }\end{array}$ & $\begin{array}{l}\text { Colony } \\
\text { Elevation }\end{array}$ & $\begin{array}{l}\text { Colony } \\
\text { Surface }\end{array}$ \\
\hline 1 & $B t$ HD-1 & CW & $\mathrm{C}$ & IR & $\mathrm{F}$ & $\mathrm{W}$ \\
\hline 2 & S 5.3 & $\mathrm{CW}$ & $\mathrm{C}$ & IR & $\mathrm{F}$ & $\mathrm{W}$ \\
\hline 3 & S 8.3 & $\mathrm{CW}$ & $\mathrm{C}$ & IR & $\mathrm{F}$ & $\mathrm{W}$ \\
\hline 4 & S 12.1 & $\mathrm{CW}$ & $\mathrm{C}$ & IR & $\mathrm{F}$ & $\mathrm{W}$ \\
\hline 5 & S 14.3 & $\mathrm{CW}$ & $\mathrm{C}$ & IR & $\mathrm{F}$ & $\mathrm{W}$ \\
\hline 6 & S 15.3 & $\mathrm{CW}$ & $\mathrm{C}$ & IR & $\mathrm{F}$ & $\mathrm{W}$ \\
\hline 7 & S 21.1 & $\mathrm{CW}$ & $\mathrm{C}$ & IR & $\mathrm{F}$ & $\mathrm{W}$ \\
\hline 8 & S 22.1 & OW & $\mathrm{C}$ & IR & $\mathrm{F}$ & $\mathrm{W}$ \\
\hline 9 & S 23.1 & DW & $\mathrm{C}$ & IR & $\mathrm{F}$ & $\mathrm{W}$ \\
\hline 10 & S 24.3 & $\mathrm{CW}$ & $\mathrm{C}$ & IR & $\mathrm{F}$ & $\mathrm{W}$ \\
\hline 11 & S 25.1 & $\mathrm{CW}$ & $\mathrm{C}$ & IR & $\mathrm{F}$ & $\mathrm{W}$ \\
\hline 12 & $\mathrm{~L} 4.1$ & $\mathrm{CW}$ & $\mathrm{C}$ & IR & $\mathrm{F}$ & $\mathrm{W}$ \\
\hline 13 & L 4.3 & $\mathrm{CW}$ & $\mathrm{C}$ & IR & $\mathrm{F}$ & $\mathrm{W}$ \\
\hline 14 & L 6.3 & $\mathrm{CW}$ & $\mathrm{C}$ & IR & $\mathrm{F}$ & $\mathrm{W}$ \\
\hline 15 & L 7.1 & $\mathrm{CW}$ & $\mathrm{C}$ & $\mathrm{R}$ & $\mathrm{F}$ & $\mathrm{W}$ \\
\hline 16 & L 8.1 & $\mathrm{CW}$ & $\mathrm{C}$ & IR & $\mathrm{F}$ & $\mathrm{W}$ \\
\hline 17 & L 11.1 & OW & $\mathrm{C}$ & IR & $\mathrm{F}$ & WR \\
\hline 18 & L 12.3 & OW & $\mathrm{C}$ & IR & $\mathrm{F}$ & $\mathrm{W}$ \\
\hline 19 & L 20.3 & OW & $\mathrm{C}$ & IR & $\mathrm{F}$ & PS \\
\hline 20 & I 1.3 & $\mathrm{CW}$ & $\mathrm{C}$ & IR & $\mathrm{F}$ & $\mathrm{W}$ \\
\hline \multicolumn{2}{|c|}{ CW- Creamy white } & \multicolumn{3}{|c|}{ DW-dirty white } & \multicolumn{2}{|c|}{ OW-off white } \\
\hline & \multirow{2}{*}{\multicolumn{3}{|c|}{ IR_-irregular margin }} & \multicolumn{2}{|c|}{ R-- regular margin } \\
\hline & $\mathrm{F}$-flat elevation & \multicolumn{2}{|c|}{$\mathrm{W}$-wavy surface } & & \multicolumn{2}{|c|}{ WR_-wrinkled surface } \\
\hline
\end{tabular}


Table.3 Biochemical characterization of representative isolates of B. thuringiensis

\begin{tabular}{|c|c|c|c|c|c|c|c|c|}
\hline \multirow{3}{*}{ Sr No. } & \multirow{3}{*}{$\begin{array}{l}\text { Isolate } \\
\text { No. }\end{array}$} & \multicolumn{7}{|c|}{ Biochemical Characteristics } \\
\hline & & \multirow[t]{2}{*}{ Catalase } & \multirow[t]{2}{*}{ Urease } & \multirow[t]{2}{*}{ Indole } & \multirow{2}{*}{$\begin{array}{c}\text { Nitrate } \\
\text { Reductase }\end{array}$} & \multicolumn{2}{|c|}{ MRVP Test } & \multirow{2}{*}{$\begin{array}{c}\text { Citrate } \\
\text { test }\end{array}$} \\
\hline & & & & & & MR & VP & \\
\hline 1 & Bt HD-1 & + & + & - & + & - & + & - \\
\hline 2 & S 5.3 & + & + & - & + & - & + & - \\
\hline 3 & S 8.3 & + & + & - & + & - & + & - \\
\hline 4 & S 12.1 & + & + & - & + & + & + & - \\
\hline 5 & S 14.3 & + & + & - & + & - & + & - \\
\hline 6 & S 15.3 & + & + & - & + & - & + & - \\
\hline 7 & S 21.1 & + & - & - & + & - & + & - \\
\hline 8 & S 22.1 & + & - & - & + & - & + & - \\
\hline 9 & S 23.1 & - & - & - & + & - & + & - \\
\hline 10 & S 24.3 & - & + & + & + & + & + & - \\
\hline 11 & S 25.1 & + & + & + & + & + & + & - \\
\hline 12 & L 4.1 & + & + & - & + & + & + & - \\
\hline 13 & L 4.3 & + & + & - & + & - & + & - \\
\hline 14 & L 6.3 & + & + & - & + & + & + & - \\
\hline 15 & L 7.1 & + & + & - & + & + & + & - \\
\hline 16 & L 8.1 & + & + & - & + & + & + & - \\
\hline 17 & L 11.1 & + & + & - & + & + & + & - \\
\hline 18 & L 12.3 & + & + & - & + & - & + & - \\
\hline 19 & L 20.3 & + & + & - & + & + & + & - \\
\hline 20 & I 1.3 & + & + & - & + & - & + & - \\
\hline
\end{tabular}

$+:$ Positive test

- : Negative test

Fig.1 Isolated colonies of native isolates of $B$. thuringiensis

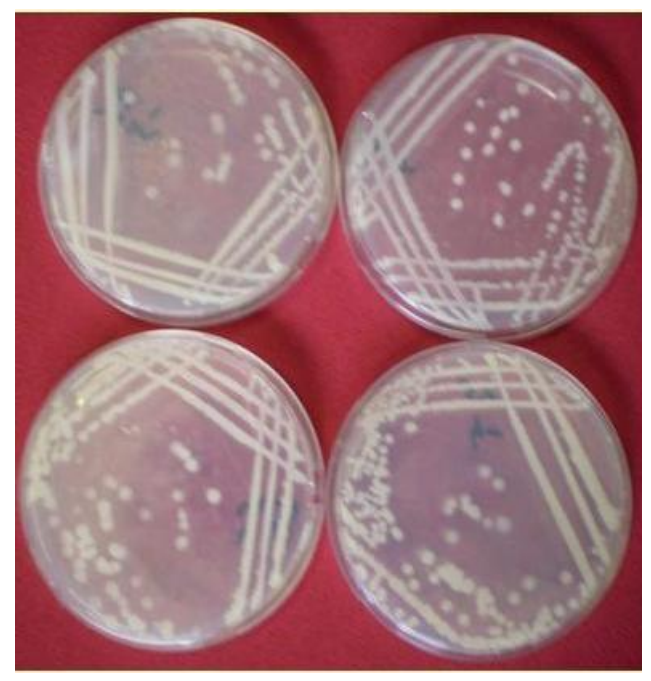


Table.4 Characterization based on staining of representative B. thuringiensis

\begin{tabular}{|c|c|c|c|c|c|c|c|c|}
\hline \multirow{3}{*}{ SN } & \multirow{3}{*}{$\begin{array}{l}\text { Isolate } \\
\text { No. }\end{array}$} & \multicolumn{7}{|c|}{ Different types of staining } \\
\hline & & \multirow{2}{*}{$\begin{array}{l}\text { Gram } \\
\text { test }\end{array}$} & \multirow[t]{2}{*}{ Ends } & \multirow{2}{*}{$\begin{array}{l}\text { Spore } \\
\text { position }\end{array}$} & \multirow{2}{*}{$\begin{array}{c}\text { Protein } \\
\text { Staining } \\
\text { (CBB) }\end{array}$} & \multicolumn{2}{|c|}{ Spore staining } & \multirow{2}{*}{$\begin{array}{l}\text { Phase } \\
\text { Contrast }\end{array}$} \\
\hline & & & & & & $\begin{array}{l}\text { Amido } \\
\text { Black }\end{array}$ & $\begin{array}{l}\text { Malachite } \\
\text { Green }\end{array}$ & \\
\hline 1 & Bt $\mathrm{HD}-1$ & + & $\mathrm{R}$ & $\mathrm{T}$ & + & + & + & + \\
\hline 2 & S 5.3 & + & $\mathrm{R}$ & $\mathrm{T}$ & + & + & + & + \\
\hline 3 & S 8.3 & + & $\mathrm{R}$ & $\mathrm{T}$ & + & + & + & + \\
\hline 4 & S 12.1 & + & $\mathrm{R}$ & $\mathrm{T}$ & + & + & + & + \\
\hline 5 & S 14.3 & + & $\mathrm{R}$ & $\mathrm{T}$ & - & - & + & + \\
\hline 6 & S 15.3 & + & $\mathrm{R}$ & $\mathrm{T}$ & + & - & + & + \\
\hline 7 & S 21.1 & + & $\mathrm{R}$ & $\mathrm{T}$ & + & + & + & + \\
\hline 8 & S 22.1 & + & $\mathrm{R}$ & $\mathrm{M}$ & - & + & + & + \\
\hline 9 & S 23.1 & + & $\mathrm{R}$ & $\mathrm{T}$ & + & + & + & + \\
\hline 10 & S 24.3 & + & $\mathrm{R}$ & $\mathrm{M}$ & - & + & + & + \\
\hline 11 & S 25.1 & + & $\mathrm{R}$ & $\mathrm{T}$ & + & + & + & + \\
\hline 12 & L 4.1 & + & $\mathrm{R}$ & $\mathrm{T}$ & + & + & + & + \\
\hline 13 & L 4.3 & + & - & $\mathrm{T}$ & + & + & + & + \\
\hline 14 & L 6.3 & + & $\mathrm{R}$ & $\mathrm{M}$ & + & + & + & + \\
\hline 15 & $\begin{array}{l}\text { L } 7.1 \\
\end{array}$ & + & $\mathrm{R}$ & $\mathrm{T}$ & + & + & + & + \\
\hline 16 & L 8.1 & + & $\mathrm{R}$ & $\mathrm{T}$ & - & - & + & + \\
\hline 17 & L 11.1 & + & $\mathrm{R}$ & $\mathrm{T}$ & - & - & - & + \\
\hline 18 & L 12.3 & + & $\mathrm{R}$ & $\mathrm{T}$ & + & + & + & + \\
\hline 19 & L 20.3 & + & $\mathrm{R}$ & $\mathrm{T}$ & - & + & + & + \\
\hline 20 & I 1.3 & + & $\mathrm{R}$ & T/M & + & + & + & + \\
\hline
\end{tabular}

$\mathrm{R}$ - Rounded ends

$\mathrm{T}$ - Terminal spore position

M - Middle spore position

+ - Positive reaction

- - Negative reaction

Fig.2 Colony characteristics of native Bt isolates

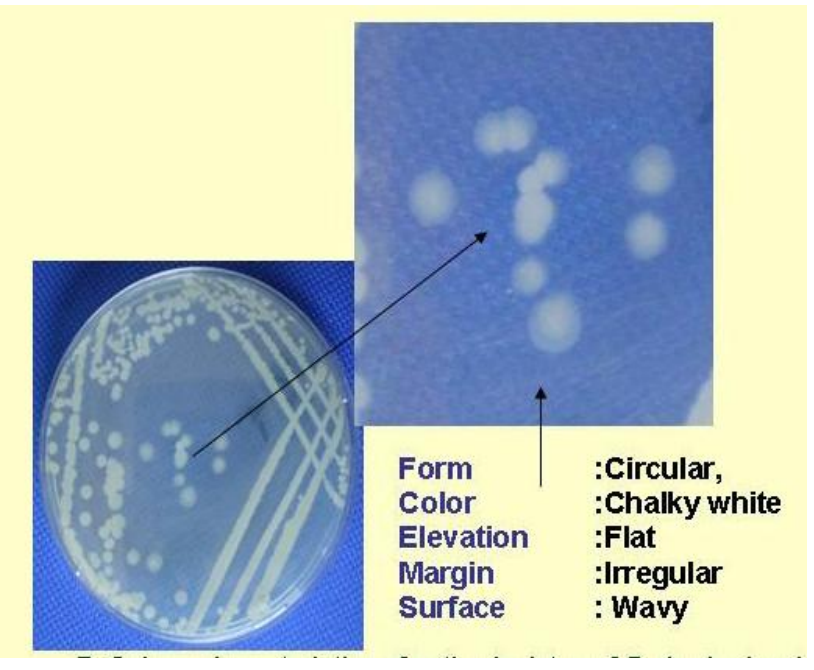


Fig.3 Biochemical characterization of native isolates of $\mathrm{Bt}$

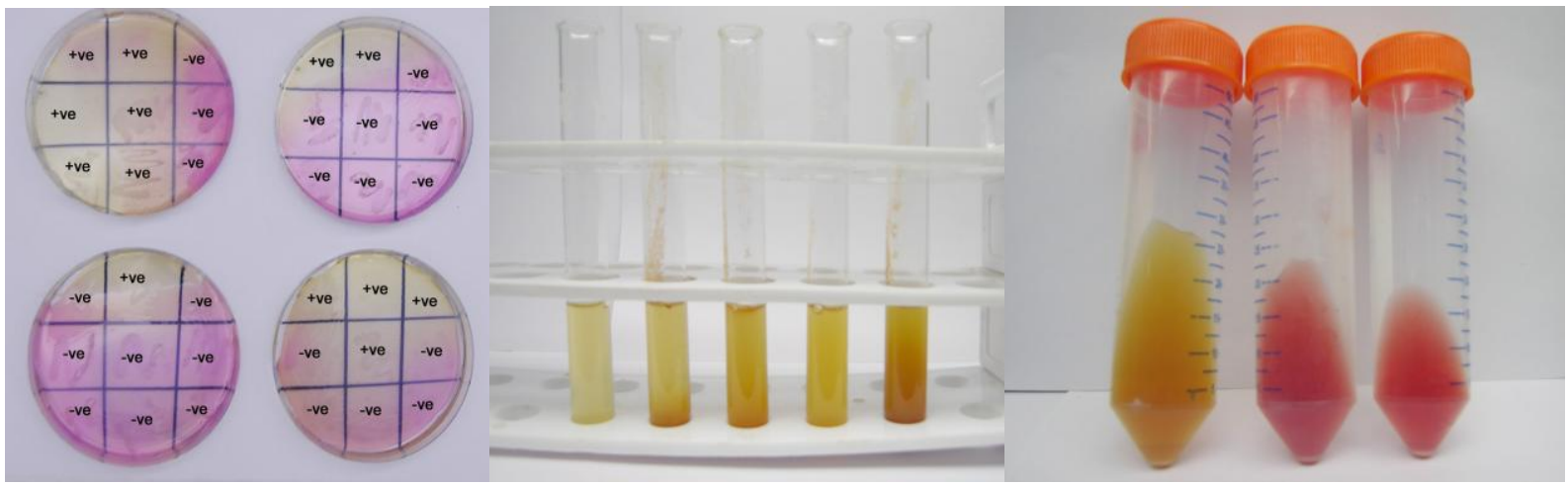

Citrate utilization test $\quad$ Nitrate reduction test

Urease test

Fig.4 a \& b Gram staining of native Bt isolates and Spore staining with Amido Black

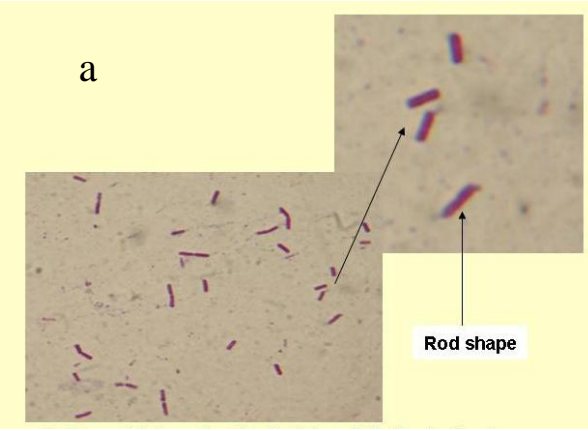

Fig.5 a \& b Spore staining with malachite green

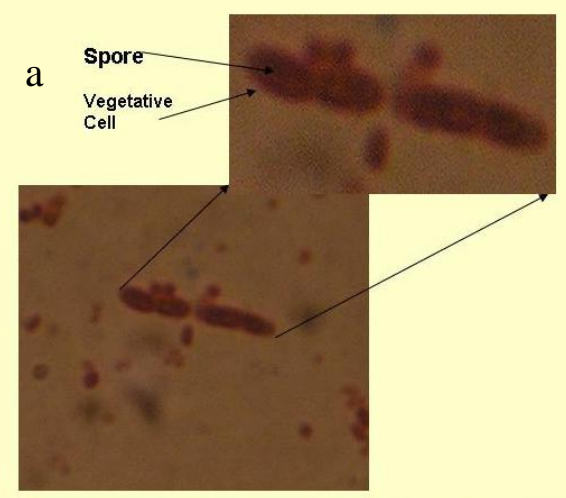

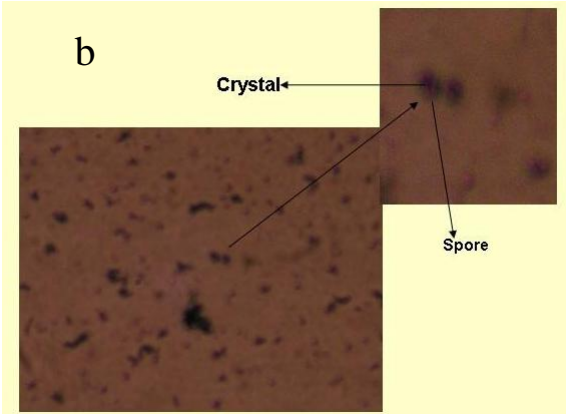

Crystal staining with CBB G-250

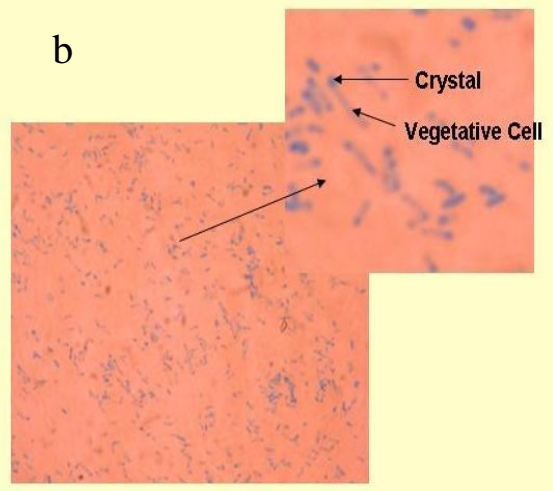


Fig.6 Phase contrast microscopic studies of native isolates of $B t$

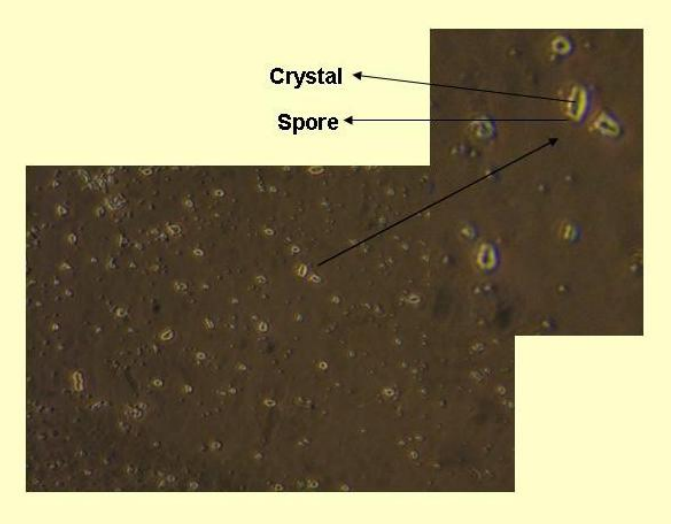

The total one hundred fifty four isolates obtained were confirmed by staining characters such as Gram reaction, whether rods or cocci with spore position, spore staining, crystal staining and phase contrast microscopy. Standard train Btk HD-1 showed positive gram test, rod shaped vegetative cells with terminal spore position and positive for spore staining with amino black and malachite green, crystal staining with commassie brilliant blue G-250 and phase contrast microscopy. Table 4 gives an idea about characterization of all the probable $\mathrm{Bt}$ isolates based on staining. Among one hundred fifty four isolates except four isolates all showed Gram positive reaction as shown in figure $4 \mathrm{a}$. Sixty nine, thirty seven and six from soil, leaf and insect cadaver respectively showed positive spore staining with amido black as shown in plate $4 \mathrm{~b}$. Seventy two, fourty seven and six isolates from soil, leaf and insect cadaver respectively showed positive spore staining with malachite green as shown in figure 5a where as eighty three, fifty five and six isolates from soil, leaf and insect cadaver respectively were positive for crystal protein staining with CBB-G250 (figure 5b). Phase contrast microscopic results revealed that there were eighty five, fifty five and six probable $B t$ isolates respectively from soil, leaf and insect cadaver as shown in figure 6 .
In conclusion, this study has given a direction to isolate new Bacillus thuringiensis from native ecological niche and also to fetch enormous genetic diversity among $\mathrm{B} t$ strains present in nature due to self transmissible nature of plasmid on which cry genes are located and transferred to the other bacillus by conjugal transfer. As insects are developing resistance against the known cry genes, identification, isolation and use of newly isolated cry genes in transgenic development is the thrust area in order to combat insect attack.

\section{References}

Allwin L., J. S. Kennedy and V. Radhakrishnan. 2007. Characterization of different geographical strains of $B t$ from Tamil Nadu. Res. J.Agri. And Biol. Sci. 3(5):362-366.

Aramideh S, Saferalizadeh MH, Pourmirza AA, Bari MR, Keshavarzi M, Mohseniazar M (2010). Characterization and pathogenic evaluation of Bacillus thuringiensis isolates from West Azerbaijan provinceIran. Afr. J. Microbiol. Res. 4(12): 1224-1229.

Asokan R. and Puttaswamy. 2007. Isolation and characterization of $B t$ Berliner from soil, leaf, seed dust and insect cadaver. 
J. Biol.Control. 20(1):83-90.

Eswarapriya B, Gopalsamy B, Kameswari B, Meera R, Devi P (2010). Insecticidal Activity of Bacillus thuringiensis IBT15Strain against Plutella xylostella. Int. J. Pharm. Tech Res. 2: 2048-2053.

Georghiou G (1990). Managing resistance to agrochemicals, from fundamental research to practical strategies. In: Green MB, Le baron HM, and Mobreg WK (eds) ACS Symposium series, 421. Am. Chem. Soc. pp 18-41.

Hernandez CS, Andrew R, Bel Y, Ferre J (2005). Isolation and toxicity of Bacillus thuringiensis from potatogrowing areas in Bolivia. J. Invertebr. Patho.1 88: 8-16.

Kati H., K. Sezen and Z. Demirbag. 2007. Characterization of a highly pathogenic $B t$ strain isolated from common cockchafer, Melolontha folia. Microbiol. 52(2):146-152.

Kaur P., N. Joshi and K. S. Brar. 2006. Morphological and biochemical characterization of $B t$ Berliner isolates and their evaluation against Plutella xylostella Linnaeus. J. Biol. Control. 20(2):191- 195.

López-Pazos, S.A. \& J. Cerón. 2007. Threedimensional structure of Bacillus thuringiensis toxins: a review. Acta Biol. Colomb. 12: 19-32.

Martin PA, Travers RS (1989). Worldwide
Abundance and Distribution of Bacillus thuringiensis Isolates. Appl. Environ. Microbiol. 55: 2437-2442.

Nester E, Thomashow L, Metz M, Gordon M (2002). 100 Years of B.thuringiensis: a Critical Scientific Assessment (online) ASM/Washington, D. C., http://www.asmusa.org.

Sarita Agrahari, M. P. Moharil, A. Pethe and N.G.V. Rao. 2008. Isolation and characterization of $B t$ strains from the ecosystem of central India. J. Pure Appl. Microbiol. 2(2):547-550.

Schnepf, E., N. Crickmore, J. van Rie, D. Lereclus, J. Baum, J. Feitelson, D.R. Zeigler \& D.H. Dean. 1998. Bacillus thuringiensis and its pesticidal crystal proteins. Microbiol. Mol. Biol. Rev. 62: 775-806.

Soberón M, Perez RV, Nuezñez-Valdéz ME, Lorence A, Gómez I, Sánchez J, Bravo A (2000). Evidence for intermolecular interaction as a necessary step for poreformation activity and toxicity of Bacillus thuringiensis Cry1Ab toxin. FEMS Microbiol. Lett. 191: 221-225.

Yamamoto T, Powell G (1993). Bacillus thuringiensis crystal proteins: recent advances in understanding its insecticidal activity. In:Kim L (ed), Advanced Engineered Pesticides. Marcel Dekker, Inc. New York, N.Y.pp. $3-42$.

\section{How to cite this article:}

Dipika Ashokrao Padole, Mangesh Pradip Moharil, Krishnananda Pralhad Ingle, Shyamkant Munje. 2017. Isolation and Characterization of Native Isolates of Bacillus thuringiensis from Vidarbha Region. Int.J.Curr.Microbiol.App.Sci. 6(1): 798-806.

doi: http://dx.doi.org/10.20546/ijcmas.2017.601.094 